\title{
[AB0 283] ARTICULAR PAIN IS CORRELATED WITH ULTRASOUND POWER DOPPLER FINDINGS?
}

\author{
D. F. Pereira', A. L. P. de Buosi ${ }^{1}$, F. B. M. D. Ferreira' ${ }^{2}$, A. Draghessi ${ }^{3}$, M. S. G. Riveros ${ }^{3}$, W. Grassi $^{3}$, J. $^{2}$ \\ Natour ${ }^{1}$, R. N. V. Furtado1. ${ }^{1}$ Rheumatology Division; ${ }^{2}$ Radiology Division, Unifesp, São Paulo, Brazil; \\ ${ }^{3}$ Rheumatology Division, Università Politecnica delle Marche, Jesi, Italy
}

Background: The presence of power Doppler (PD) gives relevant information about the inflammatory status of the synovial tissue, but there is no evidence if it is correlated with pain symptom of inflamed joints.

Objectives: To determine if there is a correlation between the presence of intra-articular PD and the pain at metacarpophalangeal (MCP) joint level of patients with RA.

Methods: Seventy-two patients with RA (based on the criteria of the American College of Rheumatology) with chronic swelling in the MCP joint and, accompanied by pain or not were recruited consecutively and assign to the painful synovitis group or the painless synovitis group. All 2 to 5 MCPs joints, bilaterally, were scanned. Clinical and US assessments were performed on the same day. The 28-joint Disease Activity (DAS 28) score; and the Stanford Health Assessment Questionnaire (HAQ) score were also performed. Inter-reader reliability of the US assessment was determined using $20 \%$ of the sample. A $5 \%$ level of significance was employed.

Results: A total of 560 MCP joints were scanned (72 patients) and that 304 MCP joints (38 patients) belonged to the painless patients group and 272 (34 patients) to the painful patients group. The painless group had 35 ( $92 \%$ ) women and the painful group had 33 (97\%). Mean age of painless patients was 60 years (range, 32-81 years) with mean disease duration of 17 years (range, 2-46 years) whereas, for painful patients, mean age was 57 years (range, 29-88 years), and mean disease duration was 15 years (range, 2-38 years). Patients in the painful group had longer morning stifness, worse DAS 28 and HAQ indexes. The majority of patients used monotherapy with a DMARD in the painless group, $44.7 \%$, versus $17.6 \%$ in the painful group, $p=0.014$; and the majority of patients in the painful group used biologic (44.1\%) as the main form of treatment versus $26.3 \%$ in the painless group, $\mathrm{p}=0.113$. In the painless group were visualized synovitis in $193(63 \%)$ MCP joints, by US-GS; PD was positive in 88 (29\%) of MCPs joints; and erosion were detected in 215 (71\%) MCP joints. In the painful group US-GS synovitis was detected in 165 (61\%) of MCPs; PD was found in 67 (25\%) of MCPs joints; and 181 (67\%) had erosion. There was a positive association between swollen and GS synovitis: OR (95\% CI), 2.1 (1.5 to 3.0); and swollen and intraarticular PD, 3.1 (2.1 to 4.8), p<0.001 for both. But there wasn't association between pain and GS synovitis, 0.9 (0.6 to 1.2$), p=0.485$; and pain and intra-articular PD, 0.8 (0.6 to 1.2$), p=0.244$.

Conclusions: Intra-articular PD wasn't associated with pain symptom in this study.

References:

Wakefield RJ et. Power-Doppler Sonography: Improving Disease Activity Assessment in Inflammatory

Musculoskeletal Disease. Arthritis \& Rheumatism 2003.

Dougados $\mathrm{M}$ et al. The ability of synovitis to predict structural damage in rheumatoid arthritis: a comparative study between clinical examination and ultrasound. Ann Rheum Disease 2012.

Disclosure of Interest: None declared

DOI: $10.1136 /$ annrheumdis-2014-eular.2127

Citation: Ann Rheum Dis 2014;73(Suppl2): 898

Session: Rheumatoid arthritis - prognosis, predictors and outcome 